\title{
Current conservation in two-dimensional ac transport
}

\author{
Jian Wang and Qingrong Zheng \\ Department of Physics, The University of Hong Kong, Pokfulam Road, Hong Kong
}

Hong Guo

Centre for the Physics of Materials, Department of Physics, McGill University, Montreal, Quebec, Canada H3A 2T8

(Received 28 August 1996; revised manuscript received 1 November 1996)

\begin{abstract}
The electric current conservation in a two-dimensional quantum wire under a time-dependent field is investigated. Such a conservation is obtained as the global density of states contribution to the emittance is balanced by the contribution due to the internal charge response inside the sample. However when the global partial density of states is approximately calculated using scattering matrix only, correction terms are needed to obtain precise current conservation. We have derived these corrections analytically using a specific two-dimensional system. We found that when the incident energy $E$ is near the first subband, our result reduces to the onedimensional result. As $E$ approaches to the $n$th subband with $n>1$, the correction term diverges. This explains the systematic deviation to precise current conservation observed in a previous numerical calculation. [S0163-1829(97)01115-6]
\end{abstract}

\section{INTRODUCTION}

The dynamic conductance of a quantum coherent mesoscopic system under a time-dependent external field is the subject of recent interests. ${ }^{1-5}$ In contrast to dc transport in the linear regime where the internal potential distribution inside the sample does not appear explicitly, the ac response depends sensitively on the internal potential distribution. This internal potential is due to the charge distribution generated by the applied external ac field at the leads and it has to be determined self-consistently. ${ }^{1}$ So far there are two approaches to the coherent ac-transport problem. One is to derive a formal linear response to a given potential distribution in the sample. ${ }^{6}$ The difficulty with such an approach is that the potential distribution is not known a priori. Another approach is to investigate the ac response to an external perturbation which prescribes the potentials in the reservoirs only. ${ }^{7,1}$ The external potentials effectively determine the chemical potential of the reservoirs and the potential distribution in the conductor must be considered a part of the response which is to be calculated self-consistently. In this approach, Büttiker and his co-workers ${ }^{1,8}$ have formulated a current conserving formalism for the low frequency admittance of mesoscopic conductors.

In the theory of Büttiker, Prêtre, and Thomas, ${ }^{1}$ it is necessary to consider the Coulomb interactions between the many charges inside the sample, in order to preserve the current conservation. For a multiprobe conductor the low frequency admittance is found to have the form ${ }^{8,9}$ $G_{\alpha \beta}(\omega)=G_{\alpha \beta}(0)-i \omega E_{\alpha \beta}+O\left(\omega^{2}\right)$, where $G_{\alpha \beta}(0)$ is the dc conductance, $E_{\alpha \beta}$ is the emittance, ${ }^{8}$ and $\alpha$ (or $\beta$ ) labels the probe. The emittance $E_{\alpha \beta}$ describes the current response at probe $\alpha$ due to a variation of the electrochemical potential at probe $\beta$ to the leading order with respect to frequency $\omega$. It can be written as ${ }^{8} E_{\alpha \beta}=d N_{\alpha \beta} / d E-D_{\alpha \beta}$, where the term $d N_{\alpha \beta} / d E$ is the global partial density of states ${ }^{10}$ (GPDOS) that is related to the scattering matrix. It describes the density of states of carriers injected in probe $\beta$ reaching probe $\alpha$ and is a purely kinetic term. The term $D_{\alpha \beta}$ is due to the Coulomb interaction of electrons inside the sample and is a term of capacitive nature. $D_{\alpha \beta}$ can be computed from the local density of states ${ }^{1,8}$ which is related to the electron dwell times. Electric current conservation, namely, $\Sigma_{\alpha} G_{\alpha \beta}(\omega)$ $=0$, means that $\Sigma_{\alpha} E_{\alpha \beta}=0$ or equivalently ${ }^{1,11}$

$$
\frac{d N_{\beta}}{d E} \equiv \sum_{\alpha} \frac{d N_{\alpha \beta}}{d E}=\sum_{\alpha} D_{\alpha \beta}=\frac{\tau_{d, \beta}}{h},
$$

where $d N_{\beta} / d E$ is the DOS and $\tau_{d, \beta}$ is the dwell time for particles coming from the probe $\beta$. Clearly the current conservation is established since one realizes that $\Sigma_{\alpha} d N_{\alpha \beta} / d E$ is the physical quantity called injectance which is identical ${ }^{8}$ to $\Sigma_{\alpha} D_{\alpha \beta}$.

Applying the above formalism to mesoscopic conductors, one needs to compute various physical quantities ${ }^{5}$ such as the partial density of states. These quantities have vivid physical meaning ${ }^{5}$ but are not easy to obtain exactly. For a large system, the GPDOS can be expressed approximately in terms of the energy derivative of the scattering matrix elements: ${ }^{12}$

$$
\frac{d N_{\alpha \beta}}{d E}=\frac{1}{4 \pi i}\left(s_{\alpha \beta}^{\dagger} \frac{d s_{\alpha \beta}}{d E}-\frac{d s_{\alpha \beta}^{\dagger}}{d E} s_{\alpha \beta}\right) .
$$

Because for a given system one may be able to obtain the scattering matrix, Eq. (2) thus provides a practical means of computing the GPDOS. On the other hand, in order to obtain current conservation precisely, a correction should be added to Eq. (2) which can be neglected for large systems and large energies. ${ }^{10,13}$ For one-dimensional (1D) systems, such a correction has been derived by Gasparian et al. ${ }^{13}$ which contains the reflection amplitude divided by the energy,

$$
\frac{d N_{\alpha}}{d E}=\frac{d \bar{N}_{\alpha}}{d E}+\operatorname{Im}\left\{\frac{s_{\alpha \alpha}}{4 \pi E}\right\},
$$

where $d \bar{N}_{\alpha} / d E \equiv \Sigma_{\beta} d N_{\alpha \beta} / d E$, which is computed from Eq. (2). 
We have recently applied the above current conserving formalism to a two-dimensional mesoscopic conductor in the shape of a $T$ junction..$^{5}$ Among other things, an interesting and we believe useful finding was that the correct $1 \mathrm{D}$ result of Eq. (3) turned out to be inadequate in 2D. First of all, by extending the results of the 1D model considered in Refs. 10,13 to the 2D case, the energy $E$ in the second term on the right hand side of Eq. (3) has to be interpreted as the longitudinal part of the incident energy. Even with this interpretation, there were small but systematic deviations to precise current conservation when the energy is approaching the second subband. In fact it was found that the DOS $d \bar{N}_{\alpha} / d E$ as defined above diverges near the onset of the second subband and this led to the observed systematic deviations. ${ }^{5}$

We are not aware of any 2D theory to account for the correction term which should appear in Eq. (3). The purpose of this paper is to investigate such correction terms in two dimensions. This not only provides further theoretical insights to the problem of ac transport, but is also helpful from a practical application point of view. From our own experience, numerical ac-transport calculations can be quite tricky and being able to obtain precise electric current conservation often serves as a very stringent check to numerical results. To this purpose, we have considered the simplest twodimensional model which is a $\delta$ potential inside a quasi-onedimensional ballistic conductor. ${ }^{14,15}$ Since quantum scattering in this system leads to mode mixing which is the basic feature of a two-dimensional system, it provides answers to our 2D problem. The advantage of this system is that it can be solved in a closed form. We have thus derived analytically the correction term. In particular, we found that when the incident energy $E$ is within the first subband, our result essentially reduces to the one-dimensional result Eq. (3). As $E$ is increased to approach the $n$th subband edge with $n>1$, the correction term diverges. This gives an explanation to the systematic deviation observed in our previous numerical calculation. ${ }^{5}$

The paper is organized as follows. In the next section we present the solution of the $2 \mathrm{D}$ scattering problem and derive the correction term. Numerical evaluations of the various quantities are also presented. The last section serves as a summary.

\section{MODEL AND RESULTS}

Figure 1 shows the system where a $\delta$ potential is confined inside a quasi-one-dimensional wire with width $a$. We assume, for simplicity of the calculation, that the boundaries of the ballistic conductor are hard walls, i.e., the potential $V=\infty$. Inside the conductor, the potential is zero except that a $\delta$ function potential $V(x, y)=\gamma \delta(x) \delta\left(y-y_{0}\right)$ is placed at $\vec{r}=\left(0, y_{0}\right)$. The scattering region $x_{1}<x<x_{2}$ is assumed to be symmetric with $x_{2}=-x_{1}=L / 2$. From now on we set $\hbar=1$ and $m=1 / 2$ to fix our units.

To compute the transmission and reflection amplitudes thus the scattering matrix, a mode matching method ${ }^{16,17,14}$ is employed. The electron wave functions are written as follows. For the region to the left of the scatterer [region I, see Fig. 1],

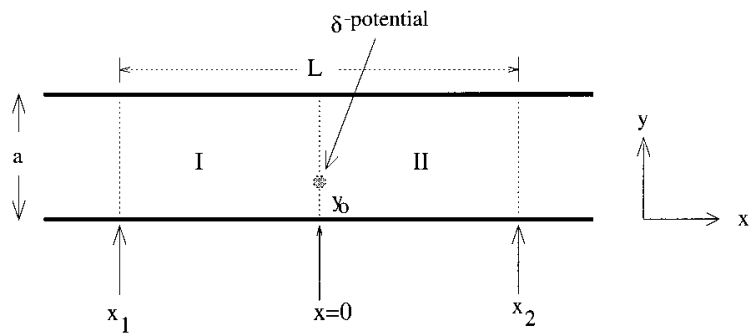

FIG. 1. Schematic plot of the quantum wire system: a $\delta$ potential $\gamma \delta\left(\vec{r}-\vec{r}_{0}\right)$ is confined inside a quasi-one-dimensional quantum wire, with $\vec{r}_{0}=\left(0, y_{0}\right)$. The wire width is $a$. The scattering region is between $x_{1}$ and $x_{2}$, where $x_{2}=-x_{1}=L / 2$. In our calculations, the parameters are set to $L=a=1, y_{0}=0.3$, and $\gamma=-1.0$.

$$
\Psi_{I}=\sum_{n} \chi_{n}(y)\left(a_{n} e^{i k_{n} x}+b_{n} e^{-i k_{n} x}\right),
$$

where $\chi_{n}(y)$ is the wave function of the $n$th subband along the $y$ direction; $a_{n}$ is the incoming wave amplitude and taken as an input parameter; $b_{n}$ is the reflection amplitude; and $k_{n}$ is the longitudinal momentum for the $n$th mode given by $k_{n}^{2}=E-(n \pi / a)^{2}$. Note that for electron traveling in the first subband, $k_{n}$ with $n>1$ is purely imaginary. Similarly for region II which is to the right of the scatterer,

$$
\Psi_{I I}=\sum_{n} \chi_{n}(y)\left(c_{n} e^{i k_{n} x}+d_{n} e^{-i k_{n} x}\right),
$$

where $c_{n}$ is transmission amplitude and $d_{n}$ is set to zero in our calculation. After matching the boundary conditions at $x=0$, we obtain

$$
a_{n}+b_{n}=c_{n}
$$

and

$$
i k_{n} c_{n}-i k_{n}\left(a_{n}-b_{n}\right)=\sum_{m} \Gamma_{n m}\left(a_{m}+b_{m}\right),
$$

where $\Gamma_{n m}=\gamma \chi_{n}^{*}\left(y_{0}\right) \chi_{m}\left(y_{0}\right)$. Eliminating $c_{n}$, we have

$$
\vec{e}=P \vec{b}
$$

where $e_{n}=-\Sigma_{m} \Gamma_{n m} a_{m}$ and $P_{n m}=\Gamma_{n m}-2 i k_{n} \delta_{n m}$. To find $\vec{b}$ we need to compute $P^{-1}$. Introducing a new matrix $\widetilde{P} \quad$ with $\quad \widetilde{P}_{n m} \equiv-P_{n m} /\left(2 i k_{m}\right)=\delta_{n m}+i \Gamma_{n m} /\left(2 k_{m}\right)=\delta_{n m}$ $+M_{n m}$, where $M_{n m} \equiv i \Gamma_{n m} /\left(2 k_{m}\right)$. Expanding $\widetilde{P}^{-1}$ in powers of $M$, we have

$$
\widetilde{P}^{-1}=\frac{1}{I+M}=I-M+M^{2}-M^{3} \cdots .
$$

From $\Gamma_{n m}=\gamma \chi_{n}^{*}\left(y_{0}\right) \chi_{m}\left(y_{0}\right)$ which is separable for index $n$ and $m$, we have

$$
\Gamma_{n m} \Gamma_{m l}=\Gamma_{n l} \Gamma_{m m} .
$$

We find that

$$
\left(M^{2}\right)_{n m}=\sum_{l} \frac{i \Gamma_{n l}}{2 k_{l}} \frac{i \Gamma_{l m}}{2 k_{m}}=\frac{i \Gamma_{n m}}{2 k_{m}} \sum_{l} \frac{i \Gamma_{l l}}{2 k_{l}}=M_{n m}(\alpha-1),
$$


where we have used Eq. (5) and $\alpha \equiv 1+i \Sigma_{n} \Gamma_{n n} /\left(2 k_{n}\right)$. Equation (6) is equivalent to $M^{2}=(\alpha-1) M$, from which we have $\widetilde{P}^{-1}=1-M+(\alpha-1) M-(\alpha-1)^{2} M+\ldots=1-M / \alpha$. Since $P_{n m}=\widetilde{P}_{n m}\left(-2 i k_{m}\right)$, we have $P=\widetilde{P} K$, where $K_{n m}$ $=-2 i k_{n} \delta_{n m}$. Hence $P^{-1}=K^{-1} \widetilde{P}^{-1}$, where $K_{n m}^{-1}=i /$ $\left(2 k_{n}\right) \delta_{n m}$. Finally, we obtain the matrix elements,

$$
\begin{aligned}
\left(P^{-1}\right)_{n m} & =\sum_{l}\left(K^{-1}\right)_{n l}\left(\widetilde{P}^{-1}\right)_{l m}=\sum_{l} \frac{i}{2 k_{n}} \delta_{n l}\left(\delta_{l m}-\frac{i \Gamma_{l m}}{2 k_{m} \alpha}\right) \\
& =\frac{i}{2 k_{n}}\left(\delta_{n m}-\frac{i \Gamma_{n m}}{2 k_{m} \alpha}\right) .
\end{aligned}
$$

We shall specialize to consider the incident electron being in the first subband: $a_{n}=\delta_{n 1}$ or $e_{n}=-\Gamma_{n 1}$. Using Eqs. (4) and (7) the reflection and transmission amplitudes are

$$
\begin{aligned}
b_{n} & =\sum_{m}\left(P^{-1}\right)_{n m} e_{m}=\sum_{m} \frac{i}{2 k_{n}}\left(\delta_{n m}-\frac{i \Gamma_{n m}}{2 k_{m} \alpha}\right)\left(-\Gamma_{m 1}\right) \\
& =\frac{i}{2 k_{n}}\left(-\Gamma_{n 1}+\sum_{m} \frac{i \Gamma_{m m}}{2 k_{m}} \frac{\Gamma_{n 1}}{\alpha}\right)=-\frac{i}{2 k_{n}} \Gamma_{n 1}\left(1-\frac{\alpha-1}{\alpha}\right) \\
& =\frac{-i \Gamma_{n 1}}{2 k_{n} \alpha}
\end{aligned}
$$

$$
c_{n}=\delta_{n 1}+b_{n} .
$$

For our system the scattering matrix elements $s_{\alpha \beta}$ are given by $s_{11}=b_{1} \exp \left(i k_{1} L\right)$ and $s_{12}=c_{1} \exp \left(i k_{1} L\right)$. The approximate DOS becomes, using Eq. (2),

$$
\begin{aligned}
\frac{d \bar{N}_{\alpha}}{d E} & =\frac{1}{4 \pi i} \sum_{\beta}\left(s_{\alpha \beta}^{\dagger} \frac{d s_{\alpha \beta}}{d E}-\frac{d s_{\alpha \beta}^{\dagger}}{d E} s_{\alpha \beta}\right) \\
& =\frac{L}{4 \pi k_{1}}-\operatorname{Im}\left(\frac{b_{1}}{4 \pi k_{1}^{2}}\right)-\frac{1}{4 \pi} \sum_{n} \frac{\left|b_{n}\right|^{2}}{i k_{1} k_{n}} .
\end{aligned}
$$

To derive this expression we have used a relation $2 b_{1}^{*}+1=\alpha / \alpha^{*}$, which follows directly from the unitary condition of the scattering matrix. Next we compute the dwell time and hence the precise DOS [as opposed to the approximate DOS of Eq. (10)]:

$$
\begin{aligned}
\tau_{d, 1} & =\frac{1}{v} \int_{I}\left|\Psi_{I}\right|^{2} d x d y+\frac{1}{v} \int_{I I}\left|\Psi_{I I}\right|^{2} d x d y \\
& =\frac{L}{2 k_{1}}+\operatorname{Re}\left(b_{1} \frac{e^{i k_{1} L}-1}{2 i k_{1}^{2}}\right)+\sum_{n}\left|b_{n}\right|^{2} \frac{e^{i k_{n} L}-1}{2 i k_{1} k_{n}},
\end{aligned}
$$

where $v=\hbar k_{1} / m$ is the velocity of the carriers at the Fermi energy. From Eqs. (1), (10), and (11), we arrive at the following central result of this work:

$$
\frac{d N_{\alpha}}{d E}=\frac{d \bar{N}_{\alpha}}{d E}+\operatorname{Im}\left\{\frac{s_{\alpha \alpha}}{4 \pi k_{1}^{2}}\right\}+\frac{1}{4 \pi} \sum_{n=2} \frac{\left|b_{n}\right|^{2}}{i k_{1} k_{n}} e^{i k_{n} L} .
$$

Hence we found that for this 2D system, there are two correction terms to the DOS. Clearly the first correction term, i.e., the second term on the right hand side of Eq. (12), is generic, as it can be written in terms of the scattering matrix element. This term is similar to the corresponding term in Eq. (3) of the 1D case, except that the total energy $E$ in Eq. (3) is now interpreted as the transport energy $k_{1}^{2}$. In fact this term has been guessed in our earlier work. ${ }^{5}$ There is a second correction term [the third term of Eq. (12)], which comes solely due to mode mixing in our $2 \mathrm{D}$ system, and understandably it does not exist in 1D cases. ${ }^{13}$

For small incident energies, i.e., as $k_{1}$ goes to zero, $\left|b_{n}\right|^{2} \rightarrow k_{1}^{2}$ for $n>1$. Therefore the second correction term of Eq. (12) is actually negligible at small energies. Indeed, this is the case in our earlier numerical calculations ${ }^{5}$ where current conservation was very well satisfied at low energies using Eq. (3). However, as the energy is approaching the $n$th subband edge, for small $k_{n} \rightarrow 0$ with $n>1,\left|b_{n}\right|^{2}$ remains finite. Hence according to Eq. (12) the second correction term diverges at these higher subband edges. This explains the observation of our calculation ${ }^{5}$ where systematic numerical errors exist in current conservation near the second subband edge. For energies within the first subband, as mentioned above $k_{n}$ are all pure imaginary numbers with $n>1$. Hence with large system size $L$, the factor $\exp \left(i k_{n} L\right)$ is very small as long as $k_{n} \neq 0$. However we emphasize that the second correction term becomes dominant very near each subband edge thus must be included in order to obtain precise current conservation.

With the analysis discussed so far, the emittance $E_{\alpha \beta}$ can be written in a closed form. However to obtain the values of $E_{\alpha \beta}$ as a function of energy some numerical computation on various summations are needed. We have evaluated the quantity $D_{\alpha, \beta}$ using the Thomas-Fermi approximation ${ }^{1,8}$ which is more appropriate to a metallic conductor,

$$
D_{\alpha, \beta}=\int d^{3} r \frac{[d n(\alpha, \vec{r}) / d E][d n(\vec{r}, \beta) / d E]}{d n(\vec{r}) / d E},
$$

where the local density of states $d n(\vec{r}, \beta) / d E$ is the injectivity which measures the additional local charge density brought into the sample at point $\vec{r}$ by the oscillating chemical potential at probe $\beta$. The injectivity can be expressed in terms of the scattering wave function, ${ }^{1}$

$$
\frac{d n(\vec{r}, \beta)}{d E}=\sum_{n} \frac{\left|\Psi_{\beta n}(\vec{r})\right|^{2}}{2 \pi v_{\beta n}}
$$

where $v_{\beta n}$ is the velocity of carriers at the Fermi energy at mode $n$ in probe $\beta . d n(\alpha, \vec{r}) / d E$ is the emissivity which describes the local density of states of carriers at point $\vec{r}$ which are emitted by the conductor at probe $\alpha$. It has been shown ${ }^{9}$ that in the absence of magnetic field the injectivity is equal to the emissivity. Using Eqs. (10), (13), and (14), we can calculate the emittance. 


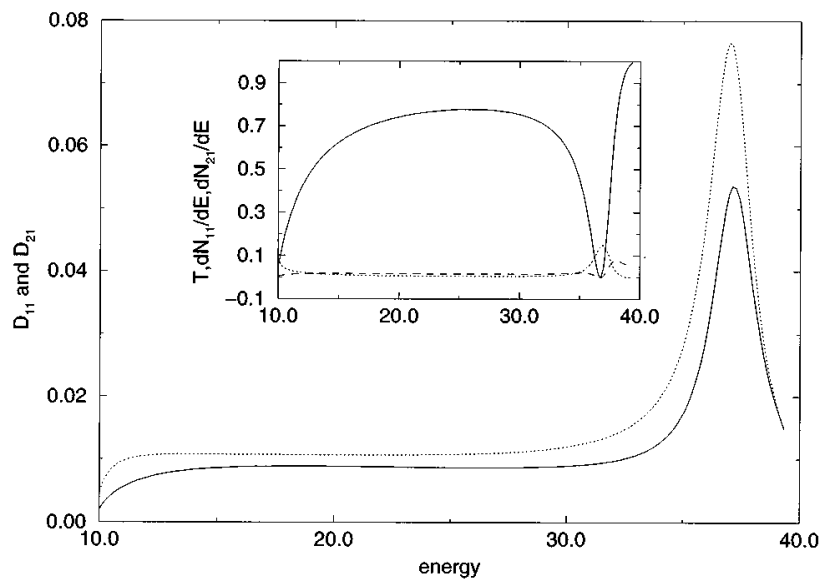

FIG. 2. The current response to the internal potential, $D_{\alpha \beta}$, computed from Eq. (13) as a function of energy $E$. Solid line: $D_{11}$; dotted line: $D_{21}$. Inset: the global partial density of states and the transmission coefficient as functions of electron energy $E$. Solid line: transmission coefficient $T(E)$; dotted line: $d N_{11} / d E$; dashed line: $d N_{21} / d E$. Unit of energy is $\hbar^{2} / 2 m a^{2}$.

Specifically, for the system of Fig. 1 we consider an incident electron coming from the left (probe 1). For this system, there is a quasibound state at an energy $E_{r}$ which is characterized by the complete reflection when the strength of the $\delta$-function potential $\gamma$ is negative. $E_{r}$ decreases as $|\gamma|$ increases. For the purpose of illustration, we have set $a=L=1, y_{0}=0.3$, and $\gamma=-1$ and found that resonant behavior occurs at $E_{r}=36.67$. Although using this set of parameters, we emphasize that the analytical result Eq. (12) is valid for both positive and negative $\gamma$.

In the inset of Fig. 2, we plot the global DOS together with the transmission coefficient $T$ as functions of energy $E$. As expected, the transmission coefficient $T(E)$ (solid line) has large values for almost all $E$ except at $E_{r}$ where the reflection coefficient $R\left(E_{r}\right)=1$. This can also be seen from the behavior of the global partial DOS for reflection $d N_{11} / d E$ (dotted line) which peaks when $T\left(E=E_{r}\right)=0$. On the other hand, $d N_{21} / d E$ (dashed line), which is the global partial DOS for transmission, takes the minimum value at $E=E_{r}$. This behavior is consistent with that of a 1D system made of a symmetric scatterer, ${ }^{10}$ where one has $d N_{11} / d E \sim R d N / d E$ and $d N_{21} / d E \sim T d N / d E$. The quantities $D_{11}$ and $D_{21}$ are shown as the solid and dotted lines in Fig. 2. Both curves reach maximum values near the resonant point $E_{r}$, which is expected since $D_{\alpha \beta}$ are proportional to the dwell time or the DOS. The emittance $E_{\alpha \beta}$ is plotted in Fig. 3. Both $E_{11}$ (solid line) and $E_{12}$ (dotted line) reach extremal values at the resonant point. The system responds differently for different energy, either capacitively when $E_{11}=-E_{12}>0$, or inductively otherwise. Figure 3 shows these responses clearly as the energy is varied. The capacitive behavior at the $T \approx 0$ resonance is the same as that observed in the 2D $T$ junction. ${ }^{5}$ On the other hand, for a $1 \mathrm{D}$ tunneling system ${ }^{1}$ the response is inductive at its resonance. But in that case the resonance is marked by the transmission coefficient being near unity.

Finally, to confirm electric current conservation, essentially the two curves of Fig. 3 must add to zero. Clearly these curves do not cancel each other as the figure shows, exactly

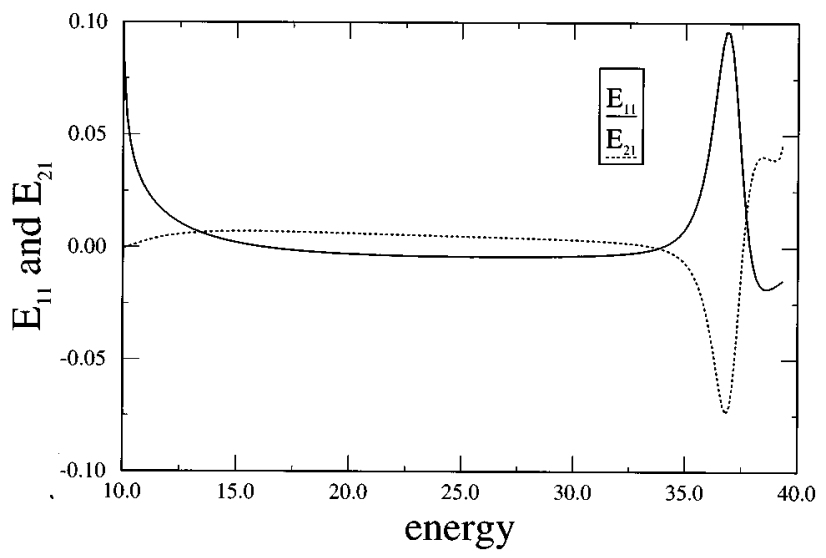

FIG. 3. The dynamic part of the admittance, $E_{\alpha \beta}$ $\equiv d N_{\alpha \beta} / d E-D_{\alpha \beta}$ as a function of energy. Solid line: $E_{11}$; dotted line: $E_{21}$.

due to the approximate nature of the partial density of states as obtained using Eq. (2) for the finite scattering volume. After including the two corrections to the DOS as derived in Eq. (12), however, we did obtain a perfect current conservation for the whole energy range. This is not surprising since after all Eq. (12) is an exact result for this quantum system.

\section{SUMMARY}

In summary, we have investigated the electric current conservation in a two-dimensional ballistic conductor under a time-dependent field. Similar to that of the 1D case, we found that in order to obtain precise current conservation, certain corrections to the density of states as obtained approximately from the scattering matrix must be included. We have derived these corrections analytically for a specific twodimensional system and found that there are two correction terms. One of the correction terms has the same form as that of the 1D case, while the second correction term is purely due to mode mixing characteristic of 2D quantum scattering. In particular, when the incident energy $E$ is within the first subband, our result essentially reduces to the onedimensional result if $E$ is not too high. On the other hand as $E$ approaches to the $n$th subband with $n>1$, the correction term diverges at the subband edges. Hence in $2 \mathrm{D}$ the mode mixing leads to important changes in the global density of states and must be included if precise electric current conservation is desired. Finally, the correction term found here provides a qualitative explanation for the small but systematic deviation to precise current conservation observed in our previous numerical calculations ${ }^{5}$ on a $2 \mathrm{D}$ quantum wire in the shape of the $T$ junction.

\section{ACKNOWLEDGMENTS}

We gratefully acknowledge support by a RGC grant from the Government of Hong Kong under Grant No. HKU 261/ 95P, a research grant from the Croucher Foundation, the Natural Sciences and Engineering Research Council of Canada, and le Fonds pour la Formation de Chercheurs et l'Aide à la Recherche de la Province du Québec. We thank the Computer Center of the University of Hong Kong for computational facilities. 
${ }^{1}$ M. Büttiker, A. Prêtre, and H. Thomas, Phys. Rev. Lett. 70, 4114 (1993); M. Büttiker and H. Thomas, in Quantum-Effect Physics, Electronics and Applications, edited by K. Ismail et al., IOP Conf. Proc. No. 127 (Institute of Physics and Physical Society, Bristol, 1992), p. 19; M. Büttiker, H. Thomas, and A. Prêtre, Z. Phys. B 94, 133 (1994).

${ }^{2}$ C. Bruder and H. Schoeller, Phys. Rev. Lett. 72, 1076 (1994).

${ }^{3}$ J. B. Pieper and J. C. Price, Phys. Rev. Lett. 72, 3586 (1994).

${ }^{4}$ W. Chen, T. P. Smith, M. Büttiker, and M. Shayegan, Phys. Rev. Lett. 73, 146 (1994).

${ }^{5}$ Jian Wang and Hong Guo, Phys. Rev. B 54, R11 090 (1996).

${ }^{6}$ H. U. Baranger and A. D. Stone, Phys. Rev. B 40, 8169 (1989); D. S. Fisher and P. A. Lee, ibid. 23, 6851 (1981); J. Cohen and Y. Avishai, J. Phys. Condens. Matter 7, L121 (1995).

${ }^{7}$ H. Pastawski, Phys. Rev. B 46, 4053 (1992).

${ }^{8}$ M. Büttiker, J. Phys. Condens. Matter 5, 9361 (1993).

${ }^{9}$ M. Büttiker and T. Christen, in Quantum Transport in Semicon- ductor Submicron Structures, edited by B. Kramer (Kluwer Academic Publishers, Dordrecht, 1996).

${ }^{10}$ V. Gasparian, T. Christen, and M. Büttiker, Phys. Rev. A 54, 4022 (1996).

${ }^{11}$ G. Iannaccone, Phys. Rev. B 514727 (1995).

${ }^{12}$ Y. Avishai and Y. B. Band, Phys. Rev. B 32, 2674 (1985).

${ }^{13}$ V. Gasparian et al., Phys. Rev. B 51, 6743 (1995).

${ }^{14}$ P. F. Bagwell, Phys. Rev. B 41, 10354 (1990).

${ }^{15}$ Another example of an "impurity" in a quantum wire is given by Christen and Büttiker who treated the quantum point contact in an external ac field. See, T. Christen and M. Büttiker, Phys. Rev. Lett. 77, 143 (1996).

${ }^{16}$ R. L. Schult, D. G. Ravenhall, and H. W. Wyld, Phys. Rev. B 39, 5476 (1989).

${ }^{17}$ See, for example, F. Sols, M. Macucci, U. Ravaioli, and K. Hess, Appl. Phys. Lett. 54, 350 (1989); Jian Wang, Yongjiang Wang, and Hong Guo, ibid. 65, 1793 (1994). 